SHORT REPORT

\title{
Examination of the hands: an insight into the health of a Welsh population
}

\author{
H A White, R Alcolado, J C Alcolado
}

See end of article for

authors' affiliations

Postgrad Med J 2003;79:588-589

Correspondence to:

Dr J C Alcolado,

Department of Medicine,

University of Wales

College of Medicine,

Heath Park, Cardiff' $\mathrm{CF} 14$

$4 \mathrm{XN}, \mathrm{UK}$;

Alcolado@btinternet.com

Submitted 27 January 2003

Accepted

12 February 2003

\begin{abstract}
When asked to examine a patient in a clinical examination, undergraduates and postgraduates characteristically begin with a painstaking observation of the hands. It was of concern that this is done mainly for the benefit of the examiners and that, in routine clinical practice, examination of the hands receives scant attention. The case notes of 70 general medical and surgical inpatients were reviewed and it was found that in only seven was there any mention of physical examination of the hands; in three of these "no clubbing" was the only entry. However, when the authors carefully examined the hands of 197 patients, at least one clinical sign was found in $87(44 \%)$ cases. Heberden's nodes, cigarette tar staining, and the spectrum of Dupuytren's contractures were the most common findings. In this population in the South Wales valleys, coal deposits were also commonly seen in men.
\end{abstract}

$\mathrm{T}$ he importance of performing a careful physical examination of the hands is stressed to medical students and doctors throughout their training. Standard textbooks discuss the wealth of information that may be obtained from diligent inspection of the hands ${ }^{1}$ and omitting this aspect of patient assessment is heavily penalised in both undergraduate and postgraduate examinations. Despite this, we became concerned that in routine clinical practice scant attention is paid to the hands and that most clinicians do not examine them or do not record abnormal findings. A search of the Medline, Embase, and PubMed databases failed to identify a single paper documenting the prevalence of physical findings in the hands among the general hospital inpatient population.

\section{METHODS}

In a two week period in 2002, we examined the hands of 197 patients admitted to general medical and surgical wards at the Royal Glamorgan Hospital, Llantrisant, Mid Glamorgan, UK. This is a district general hospital with 400 beds that serves a local population of 180000 . It is situated in the South Wales valleys, an area that until recently had an active coal mining industry. In order to reduce selection bias, we attempted to examine every patient on the wards, regardless of age, gender, diagnosis, or presenting complaint. Both hands were carefully inspected and any physical signs recorded. A physical sign was defined as an abnormality that was clearly present, could be demonstrated to an independent general physician or medical student, and was typical of examples shown in standard textbooks of physical examination. ${ }^{1}$ During a separate period we checked the medical notes of 70 inpatients for evidence of a documented physical examination of the hands. We excluded all cases in which the presenting complaint or diagnosis was specifically focused on the hands.

\section{RESULTS}

The 197 patients we examined (118 female) had a median age of 74 years with a mean age of 70.8 years (range 19-101 years). Eighty seven (44\%) had at least one physical sign in the hands (table 1). Coal deposition was found in 13/79 men but in none of the women, in keeping with the occupational history of our population. Smoking rates in the Welsh valleys are estimated at $30 \%^{2}$ but we found signs of this in the hands of only 10/79 (13\%) of men and 7/118 (6\%) of women. Our current sample was too small to study the possible associations with duration of smoking, type, and quantity of cigarettes or duration of abstention with tar staining of the fingers. Heberden's nodes were found in 21 / $118(18 \%)$ females but only $3 / 79(4 \%)$ males (by $\chi^{2}$ test, $\mathrm{p}<0.01)$.

We found that only $7 / 70(10 \%)$ of the inpatient notes that we reviewed had any mention of an examination of the hands. In three of the cases the absence of clubbing was documented. In the remaining four cases a physical sign was noted (one each of finger clubbing, tar staining, leukonychia, and rheumatoid changes).

Table 1 Physical findings in the hands of 197 patients

\begin{tabular}{lll}
\hline Physical sign & Frequency & Prevalence (\%) \\
\hline No physical signs & 110 & 55.8 \\
Heberden's nodes & 24 & 12.2 \\
Tar staining & 17 & 8.6 \\
Coal deposits & 13 & 6.6 \\
Dupuytren's spectrum & 13 & 6.6 \\
MCP joint swelling & 11 & 5.6 \\
Pale palms & 7 & 3.6 \\
Intrinsic muscle wasting & 6 & 3.0 \\
Boutonniere's deformity & 5 & 2.5 \\
Bouchard's nodes & 5 & 2.5 \\
Palmar erythema & 4 & 2.0 \\
Clubbing & 4 & 2.0 \\
Jaundice & 3 & 1.5 \\
Peripheral cyanosis & 2 & 1.0 \\
Ulnar deviation of fingers & 2 & 1.0 \\
Congenital contracture of fifth digit & 2 & 1.0 \\
Incomplete leukonychia & 2 & 1.0 \\
Complete leukonychia & 1 & 0.5 \\
Gouty tophi & 1 & 0.5 \\
Swan neck deformity & 1 & 0.5 \\
Z shaped thumb deformity & 1 & 0.5 \\
Median nail dystrophy & 1 & 0.5 \\
Beau's lines & 1 & 0.5 \\
\hline MCP, metacarpophalangeal. & & \\
\hline
\end{tabular}




\section{LEARNING POINTS}

- Examination of the hands should be an integral part of the general physical examination of all patients.

- Despite the importance placed on this aspect of physical examination in medical education, only $10 \%$ of general medical or surgical inpatient notes make any mention of the hands.

- Even when examination of the hands is recorded, details are often scant-for example, "no clubbing".

- Careful examination of the hands will reveal physical signs in more than $40 \%$ of patients.

- Some physical signs-for example, coal deposits or Heberden's nodes, may be a marker of previous occupation.

- Tar staining of the fingers is seen only in a minority of smokers.

\section{DISCUSSION}

The aim of physical examination is to obtain the maximum amount of reliable information with the minimum of patient inconvenience or distress in a relatively short period of time. It is customary to shake hands with a patient and we believe a careful inspection of the hands at this stage should not be neglected. Although doctors may not specifically record the absence of signs in medical records, we were concerned that the hands were not mentioned at all in $90 \%$ of medical notes. While we found $44 \%$ of patients we examined had physical signs in the hands, fewer than 6\% (4/70) of inpatient notes recorded their presence $(\mathrm{p}<0.0001)$.

The prevalence of coal staining of the hands in our population is likely to be much higher than in non-mining communities, but it serves as an important clinical reminder

of the health risks that many men in the Welsh valleys have endured. The overall prevalence and gender differences of Heberden's nodes are similar to those reported in a previous study. ${ }^{3}$ It has been suggested that this physical sign is associated with occupations requiring sustained precision grip force. ${ }^{4}$ One of our female patients, with predominantly left handed Heberden's nodes, was convinced that they were related to her previous work as a weaver and we were able to identify a case report that lends support to her contention. $^{5}$

The fact that undergraduates and postgraduates almost invariably examine the hands when undergoing a test of their clinical ability, such as finals or the MRCP, suggests they have been taught and learnt its potential clinical value. It would be interesting to ask doctors why they subsequently appear to discard this clinical skill. Given it is relatively simple, quick and reliable, we would encourage all physicians to examine the hands once again.

\section{Authors' affiliations}

H A White, J C Alcolado, Department of Medicine, University of Wales College of Medicine, Cardiff

R Alcolado, Department of Medicine, Royal Glamorgan Hospital, Ynysmaerdy, Mid Glamorgan

\section{REFERENCES}

1 Munro JF, Campbell IW, eds. MacLeod's clinical examination. 10th Ed. London: Churchill Livingstone, 2000.

2 National Assembly of Wales. Welsh health survey 1998. Cardiff: National Assembly of Wales, 1998.

3 Bergenudd $\mathbf{H}$, Lindgarde F, Nilsson B. Prevalence and coincidence of degenerative changes of the hands and feet in middle age and their relationship to occupational work load, intelligence and social background. Clin Orthop 1989;239:306-10.

4 Lawrence J. Rheumatism in cotton operators. Br J Ind Med 1961;18:270-6.

5 Turner W, Collin J. Seamstress's finger: a cause of Heberden's nodes. BMJ 1988;297:1636. 